\title{
Investigating the Natural History and Prognostic Nature of NTRK Gene Fusions in Solid Tumors
}

\section{Limin Zhu ( $\nabla$ joy801@gmail.com )}

Baylor College of Medicine https://orcid.org/0000-0002-8723-7427

\section{Brian Hobbs}

The University of Texas at Austin Dell Medical School

\section{Jason Roszik}

The University of Texas MD Anderson Cancer Center

\section{Vijaykumar Holla}

The University of Texas MD Anderson Cancer Center

\section{David S. Hong}

The University of Texas MD Anderson Cancer Center

\section{Research Article}

Keywords: TRK inhibitors, NTRK gene fusion, Solid tumors

Posted Date: June 28th, 2021

DOI: https://doi.org/10.21203/rs.3.rs-573656/v1

License: (c) (1) This work is licensed under a Creative Commons Attribution 4.0 International License. Read Full License

Version of Record: A version of this preprint was published at Investigational New Drugs on August 2nd, 2021. See the published version at https://doi.org/10.1007/s10637-021-01157-8. 
Investigating the natural history and prognostic nature of NTRK gene fusions in solid tumors Limin Zhu, MD $\mathrm{PhD}^{1}$, Brian Hobbs, $\mathrm{PhD}^{2}$, Jason Roszik, $\mathrm{PhD}^{3}$, Vijaykumar Holla, $\mathrm{PhD}^{3}$, David S. Hong, $\mathrm{MD}^{3}$

Affiliations:

${ }^{1}$ Baylor College of Medicine, Houston, TX

${ }^{2}$ The University of Texas at Austin Dell Medical School, Austin, TX

${ }^{3}$ The University of Texas MD Anderson Cancer Center, Houston, TX

Corresponding author: David S. Hong, MD, Professor, Deputy Chair, Department of Investigational Cancer Therapeutics, The University of Texas MD Anderson Cancer Center, 1515 Holcombe Blvd., Houston, TX 77030. Tel: 713-563-5844, Fax: 713-563-0566, Email: dshong@mdanderson.org 


\section{Declarations}

Funding: This study was funded by National Institute of Health and Bayer Corporation

\section{Conflicts of interests: None}

Availability of data and material: All data generated and analyzed during the current study are available from the corresponding author on reasonable request.

Code availability: Not applicable

Ethics approval: This retrospective study involving human participants was in accordance with the ethical standards of the institutional and national research committee and with the 1964 Helsinki Declaration and its later amendments or comparable ethical standards. The Human Investigation Committee (IRB) of MD Anderson Cancer Center approved this study.

Consent to participate: Informed consent was obtained from all individual participants included in the study.

Consent to publish: The authors affirm that human research participants provided informed consent for publication of the data collected. 


\section{ABSTRACT}

Background: Several TRK inhibitors have demonstrated clinical efficacy in patients with solid tumors harboring NTRK gene fusions. However, the natural history and prognostic implications of NTRK fusions in solid tumors remain unknown.

Methods: A cohort of 77 MD Anderson Cancer Center patients (MDACC) with NTRK gene fusions was identified and retrospectively compared to a second cohort from the Cancer Genome Atlas (TCGA) database. Due to paucity of events in early stage cancers and lack of TCGA data in rare tumors, 25 randomly selected MDACC patients were matched to 122 TCGA patients without NTRK gene fusion. Next we assessed the associations between NTRK gene fusion and overall (OS) and progression-free survivals (PFS).

Results: Among the 77 MDACC patients with NTRK gene fusions, 18 NTRK fusion partners were identified. There were insufficient OS events for analysis in the matched cohort. PFS was not significantly different $(\mathrm{p}=0.49)$ between the NTRK-fusion positive MDACC patients (median PFS 786 weeks, 95\% CI 317-NE) and the NTRK-fusion negative TCGA patients (median PFS NE). The adjusted hazard ratio comparing TCGA patients to MDACC patients was HR=0.72 (95\% CI: 0.23-2.33), which trended towards a reduced rate of progression or death experienced by TCGA patients.

Conclusions: This study did not identify statistically significant associations between NTRK fusion and PFS. Nonsignificant trends estimated increases in the risk of progression or death events for patients with NTRK fusions when compared to matched controls. Our findings help illuminate the influence of NTRK fusions on the natural history of a variety of solid tumors.

Keywords: TRK inhibitors, NTRK gene fusion, Solid tumors 


\section{Introduction}

Neurotrophic tyrosine receptor kinases (NTRK) 1, 2, and 3 are three distinct genes that encode the tropomyosin receptor kinase (TRK) proteins TRK A, B and C respectively [1]. Activation of TRKs promote cell proliferation, differentiation and survival [2]. NTRK gene fusions, which involve intra- or interchromosomal rearrangements of the kinase domain with various partners that lead to ligand independent activation of downstream signaling pathways, have been shown to be oncogenic drivers across a wide variety of adult and pediatric cancers [3]. NTRK gene fusions occur at frequencies ranging from $<1 \%$ in some common tumors such as lung, colorectal, pancreatic, breast cancers, and melanoma, to 5\%-25\% in papillary thyroid cancers, Spitzoid neoplasms, and gastrointestinal stromal tumors (GIST), and up to $>90 \%$ in rare tumor types including secretory breast carcinoma and secretory carcinoma of the salivary glands [4-7].

Over the past few years, several small molecule inhibitors targeting TRK fusion proteins have been developed and demonstrated safety and efficacy in clinical trials. For example, larotrectinib, a highly selective and potent pan-TRK inhibitor, has showed durable antitumor efficacy in children and adults with solid tumors harboring NTRK gene fusions [8]. The second-generation pan-TRK inhibitor selitrectinib (formerly known as LOXO-195) has also now demonstrated preliminary efficacy in patients with resistance to prior TRK inhibitors [9]. Despite the robust clinical responses to TRK inhibitors, the natural history and prognostic implications of NTRK fusions in solid tumors is poorly understood, mostly due to the rarity of such mutations. Indeed, only one study has so far evaluated the prognosis of NTRK fusions from a cohort of 27 cancer patients with NTRK fusions and 107 matched NTRK-fusion negative patients [10]. Preliminary data showed no clear difference in survival between two groups.

We have now evaluated and compared the progression-free survival between MD Anderson Cancer Center (MDACC) patients with NTRK fusions and The Cancer Genome Atlas (TCGA) patients with comparable histology but without NTRK fusions. Of note, the MDACC cohort of 77 patients with NTRK gene fusions is to date the largest such population at a single site.

\section{Materials and Methods}

\section{Patients}

A total of 77 MDACC patients with solid tumors harboring NTRK fusions were identified by various molecular profiling tools (Foundation One, Guardant 360, Oncomine, STGA 2018, CARIS, FISH, or PCR): 37 were treated with TRK inhibitors in clinical trials for larotrectinib (NCT02122913, NCT02576431) or selitrectinib (NCT03215511); the remaining 40 patients were not treated with TRK inhibitors. This cohort of 77 NTRK-fusion positive MDACC patients was retrospectively compared to a second cohort extracted from the external TCGA database. The TCGA cohort consists of 4630 patients identified as having a tumor histology observed in at least one MDACC patient [11]. Exclusion criteria removed 5 MDACC patients with insufficient follow-up for progression-free survival. Additionally, 10 MDACC and 2154 TCGA patients missing pathological stage at diagnosis were excluded from matching and statistical analysis. The remaining 62 MDACC patients harboring NTRK fusions were matched to 2476 TCGA patients (2466 patients without NTRK fusion, 10 patients with NTRK fusions 
who were later removed during matching process). Figure 1 depicts the matching process.

Supplementary table 1 describes the clinical characteristics of these patients.

\section{Matching}

\section{Matched cohort I}

Based on identical histology (TCGA code), pathological stage at diagnosis, and sex, 25 MDACC patients with NTRK fusions were matched to 407 commensurate TCGA patients (406 patients without NTRK fusions, 1 patient with NTRK fusion). Matches were identified for patients with breast invasive carcinoma (BRCA), thyroid carcinoma (THCA), colon adenocarcinoma (COAD), and pancreatic adenocarcinoma (PAAD). Of the combined 432 patients in this cohort, 126 patients were stage I, 285 patients were stage II, and 21 were stage III at initial diagnosis.

\section{Matched cohort II}

The initial matched cohort presented imbalances among the histologies, with an overrepresentation of breast cancer patients in the TCGA cohort. Therefore, a second matched cohort was derived from matched cohort I, imposing a 5:1 ratio between randomly selected TCGA patients and MDACC patients. Accordingly, patients with BRCA, THCA, COAD were reduced to 5 to 1 matches, while PAAD patients were kept at 2:1 ratio as only 3 patients were identified in this histology (2 TCGA and 1 MDACC patients). This process yielded a cohort of 147 patients including 25 MDACC and 122 TCGA patients. The one TCGA patient with NTRK fusion identified in matched cohort I was excluded in matched cohort 2 therefore all 122 TCGA patients were negative for NTRK fusions. Of the combined 147 patients, 109 patients were stage I, 26 patients were stage II, and 12 were stage III at initial diagnosis. Supplementary table 2 describes the clinical characteristics of the final matched cohort.

\section{Statistical Analyses}

Statistical analyses evaluated the endpoint progression-free survival (PFS). Progression-free survival was calculated as a composite endpoint of the duration from initial diagnosis of primary tumor to death, first progression, or start of TRK inhibitor therapy, whichever was earliest. Both progression and death were considered events. A patient was right-censored at the start of TRK inhibitor therapy. Thus, the impact of TRK inhibitor therapy is not estimated by statistical findings reported in this study.

Progression-free survival was estimated using Kaplan-Meier method with Greenwood's formula for interval estimation. We report the median follow-up with inter-quartile range lower and upper bounds. Median event durations are reported with 95\% confidence intervals (CI). The value "NE" (not estimated) is reported for interval bounds for which insufficient follow-up precludes statistical estimation. Statistical association between NTRK gene fusion and overall survival (OS) were not estimated because of the paucity of events among MDACC patients (refer to supplementary table 3).

Univariate statistical analyses were applied to test for statistical associations between progression-free survival and NTRK, NTRK.3L, SEX, STAGE, STAGE.2L, and TYPE (definitions of these variables are described in supplementary table 4). Hypothesis testing used Cox proportional hazards regression with p-values computed from the Likelihood Ratio Test. Multiple regression was implemented to adjust for stage and age at initial diagnosis. Hazard ratios (HR) are reported with 95\% CI and p-values arising 
from Wald Test. Akaike information criterion (AIC) was used to evaluate model complexity versus goodness of fit. P-values of less than 0.05 were considered statistically significant. Two-sided tests for association between PFS and NTRK obtain $80 \%$ power to detect only large statistical effect sizes, as reflected by hazard ratios of 0.4 or less. All statistical analyses were performed in R version 3.6.3 using the survival package (https://cran.r-project.org/web/views/Survival.html).

\section{Results}

\section{Baseline characteristics of NTRK-fusion positive patients}

A total of 77 cancer patients with NTRK fusions were identified at MD Anderson Cancer Center. Patients ranged from 1 year old to 84 years old, with 34 male and 44 female. These patients had thyroid cancer $(n=34)$, salivary gland cancer $(n=22)$, sarcoma $(n=5)$, CNS tumor $(n=4)$, colorectal cancer $(n=4)$, lung cancer $(\mathrm{n}=2)$, pancreatic cancer $(\mathrm{n}=2)$, breast cancer $(\mathrm{n}=2)$, cholangiocarcinoma $(\mathrm{n}=1)$, and small intestine cancer $(\mathrm{n}=1)$. Demographic characteristics, tumor type, and stage are summarized in Table 1. In this NTRK fusion-positive cohort, NTRK3 gene fusions $(67 \%)$ occurred more frequently than NTRK1 $(30 \%)$ and NTRK2 (3\%). A total of 18 different NTRK fusion partners were identified; the most frequently detected fusions were NTRK3-EVT6 $(n=43)$, NTRK1-TPM3 $(n=9)$, and NTRK1-TPR $(n=4)$ (Table 2).

\section{Influence of NTRK gene fusions on natural history of solid tumors}

Due to paucity of events in early stage cancers and lack of TCGA data in a variety of rare tumors including salivary gland cancer, 25 of the 77 MDACC patients harboring NTRK fusions were matched to 122 TCGA patients without NTRK fusions encompassing four types of solid tumors including breast cancer, thyroid cancer, colon cancer, and pancreatic cancer. Of the 25 MDACC patients, 12 and 13 had NTRK1 and NTRK3 fusions, respectively. There were insufficient OS events for analysis in the matched cohort.

Median PFS was 786 weeks (95\% CI 317-NE) for the 25 MDACC patients with NTRK fusions while median PFS could not be determined in the 122 TCGA patients without NTRK fusions (Figure 2). Statistical analysis showed no significant difference in PFS between these two cohorts with neither univariate analysis $(\mathrm{p}=0.49)$ nor multiple regression adjusting for stage and age $(\mathrm{p}=0.59)$. The adjusted hazard ratio comparing NTRK-fusion negative TCGA patients to NTRK-fusion positive MDACC patients was $\mathrm{HR}=0.72$ (95\% CI: $0.23-2.33)$, which trended towards a reduced rate of progression or death experienced by TCGA patients. Additionally, PFS was not significantly different $(\mathrm{p}=0.78)$ when accounting for NTRK fusion subtypes comprised of 12 MDACC patients with NTRK1 fusion (median PFS 317 weeks, 95\% CI 191-NE) and 13 MDACC patients with NTRK3 fusion (median PFS 786 weeks, 95\% CI 162-NE) (Figure 3). After adjusting for initial stage and age, the estimated rate of progression or death was 0.74 (95\% CI: 0.19-2.93) and 0.72 (95\% CI: 0.19-2.68) times lower for TCGA patients without NTRK fusion when compared to patients harboring NTRK1 and NTRK3 fusions, respectively. These findings were not statistically significant $(\mathrm{p}=0.665, \mathrm{p}=0.621)$. 


\section{Discussion}

Evaluating patients with multiple tumor types, this study did not identify statistically significant associations between progression-free survival and NTRK fusion. Nonsignificant statistical trends estimated increases in the risk of progression or death events for patients with NTRK fusions when compared to matched controls. In addition, we characterized the clinical and molecular features of 77 patients with solid tumors harboring NTRK fusions, to date the largest such population at a single site. Our study identified multiple novel NTRK fusions that have not been previously reported in literature. So far, the only study that evaluated the prognosis of NTRK fusions included 27 patients with NTRK fusions that encompassed 12 different types of tumors, with only 1-2 patients from each tumor type [10]. In comparison, our analysis of progression-free survival included 25 NTRK fusion-positive patients from 4 different tumor types and 21 of them had thyroid cancer which is known to be a solid tumor with relatively high frequency of NTRK fusions. Our findings help illuminate the influence of NTRK fusions on the natural history of a variety of solid tumors and reinforce the observed anti-tumor effect of TRK inhibitors.

Of note, our analysis was limited by the paucity of progression or death events in early stage cancers, by the diversity of tumor types included, and by the lack of TCGA data in rare cancers such as salivary gland cancer, which was a major histology subtype in MDACC patients. Indeed, 52 of the 77 MDACC patients with NTRK fusions were lost during the matching process to TCGA cases therefore only 25 MDACC patients were used in the final analysis. In addition, clinical outcomes of NTRK fusion positive patients in this study were captured from the electronic health records of MDACC and other relevant hospitals/clinics, thus treatments or outcomes that occurred outside the expanded record system may be missing. Moreover, the study is insufficiently powered to detect small but clinically meaningful effect sizes. Further studies will aim to identify a larger patient population with confirmed NTRK wild type tumors but similar histologies and cancer stages to improve the analysis of clinical outcomes. 


\section{References}

[1] Klein R, Jing S, Nanduri V, O'Rourke E, Barbacid M. The trk proto-oncogene encodes a receptor for nerve growth factor. Cell 1991. https://doi.org/10.1016/0092-8674(91)90419-Y.

[2] Kaplan DR, Martin-Zanca D, Parada LF. Tyrosine phosphorylation and tyrosine kinase activity of the trk proto-oncogene product induced by NGF. Nature 1991. https://doi.org/10.1038/350158a0.

[3] Rubin JB, Segal RA. Growth, survival and migration: the Trk to cancer. Cancer Treat Res 2003. https://doi.org/10.1007/0-306-48158-8_1.

[4] Brenca M, Rossi S, Polano M, Gasparotto D, Zanatta L, Racanelli D, et al. Transcriptome sequencing identifies ETV6-NTRK3 as a gene fusion involved in GIST. J Pathol 2016. https://doi.org/10.1002/path.4677.

[5] Haller F, Knopf J, Ackermann A, Bieg M, Kleinheinz K, Schlesner M, et al. Paediatric and adult soft tissue sarcomas with NTRK1 gene fusions: A subset of spindle cell sarcomas unified by a prominent myopericytic/haemangiopericytic pattern. J Pathol 2016.

https://doi.org/10.1002/path.4701.

[6] Bishop JA, Yonescu R, Batista D, Eisele DW, Westra WH. Most nonparotid "acinic cell carcinomas" represent mammary analog secretory carcinomas. Am J Surg Pathol 2013. https://doi.org/10.1097/PAS.0b013e3182841554.

[7] Prasad ML, Vyas M, Horne MJ, Virk RK, Morotti R, Liu Z, et al. NTRK fusion oncogenes in pediatric papillary thyroid carcinoma in northeast United States. Cancer 2016. https://doi.org/10.1002/cncr.29887.

[8] Drilon A, Laetsch TW, Kummar S, Dubois SG, Lassen UN, Demetri GD, et al. Efficacy of larotrectinib in TRK fusion-positive cancers in adults and children. N Engl J Med 2018. https://doi.org/10.1056/NEJMoa1714448.

[9] Hyman D, Kummar S, Farago A, Geoerger B, Mau-Sorensen M, Taylor M, et al. Abstract CT127: Phase I and expanded access experience of LOXO-195 (BAY 2731954), a selective nextgeneration TRK inhibitor (TRKi), 2019. https://doi.org/10.1158/1538-7445.sabcs18-ct127.

[10] Bazhenova L, Jiao X, Lokker A, Snider J, Castellanos E, Nanda S, et al. Abstract 09: Cancers with NTRK gene fusions: Molecular characteristics and prognosis , 2020. https://doi.org/10.1158/1557-3265.advprecmed20-09.

[11] Liu J, Lichtenberg T, Hoadley KA, Poisson LM, Lazar AJ, Cherniack AD, et al. An Integrated TCGA Pan-Cancer Clinical Data Resource to Drive High-Quality Survival Outcome Analytics. Cell 2018. https://doi.org/10.1016/j.cell.2018.02.052. 


\section{Figure 1. Flow chart of the matching process}

\section{MDACC}

Assessed for NTRK fusion ( $n=77)$

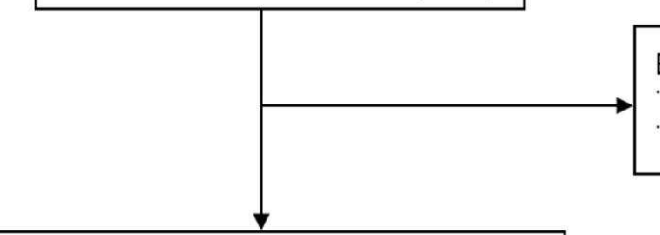

Evaluable for statistical analysis $(n=62)$

\section{Excluded $(n=15)$}

Insufficient follow-up $(n=5)$

Missing Pathological Stage $(n=10)$
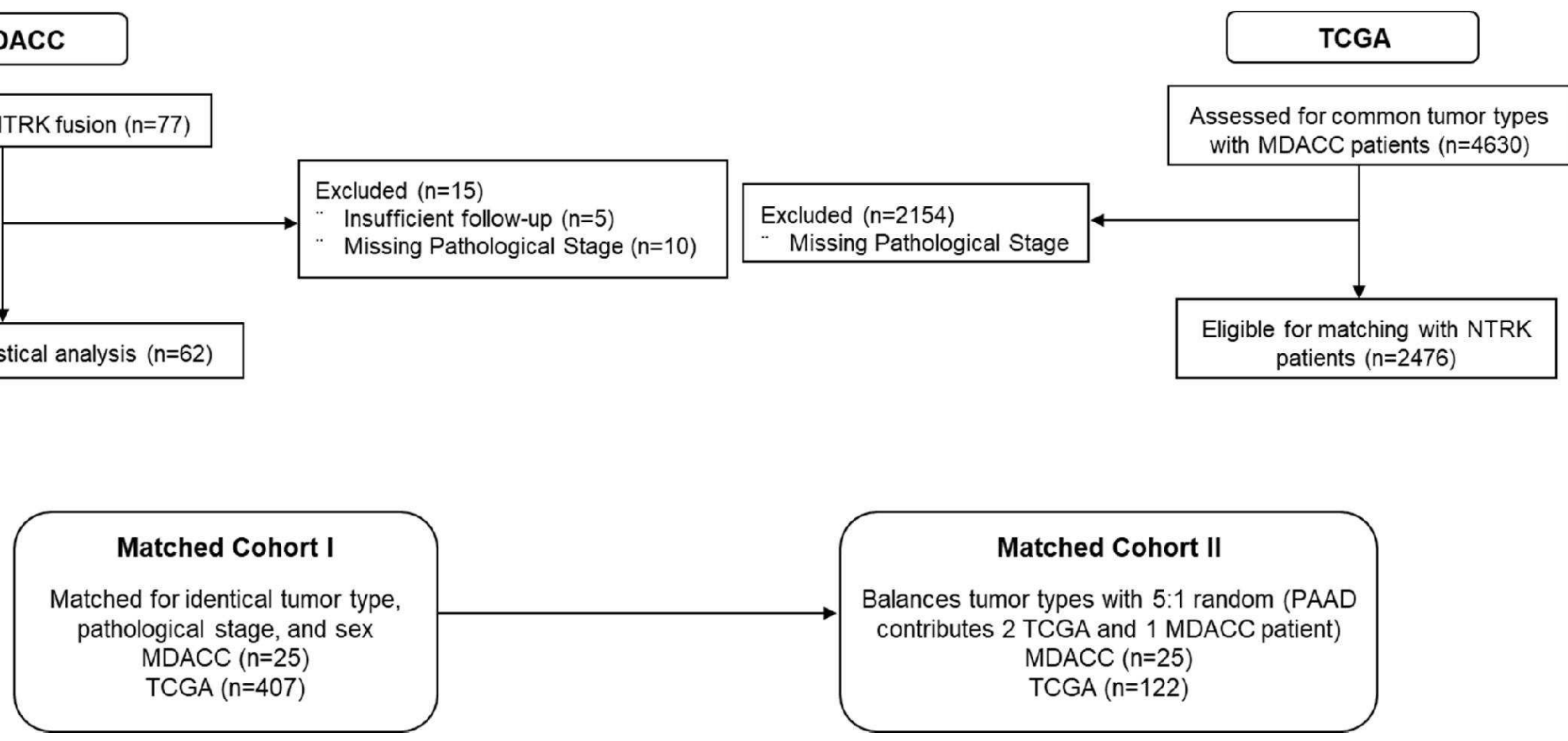
Figure 2. Kaplan-Meier PFS by NTRK status

PFS of NTRK-fusion positive vs. negative patients

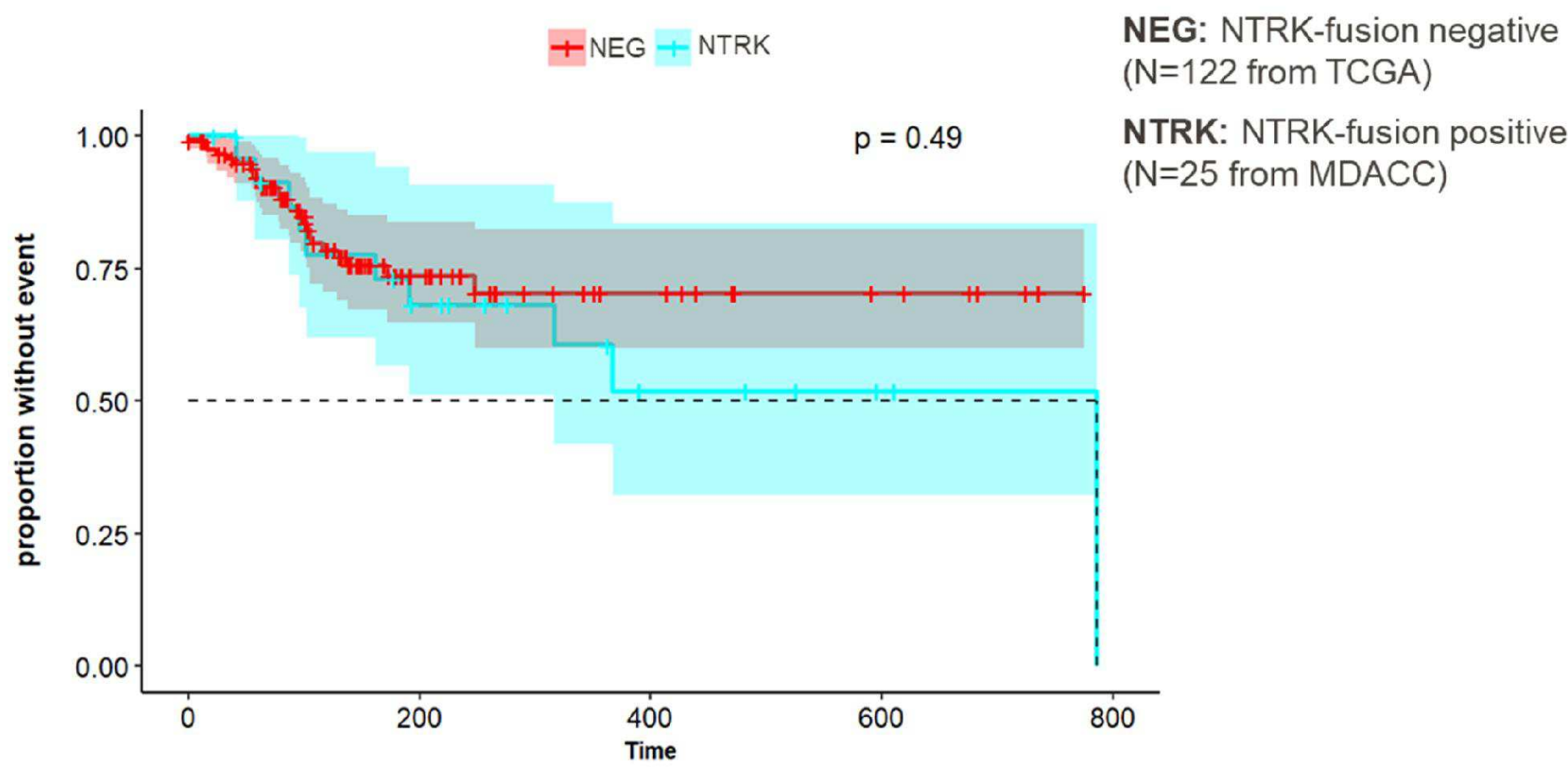

\section{Number at risk}

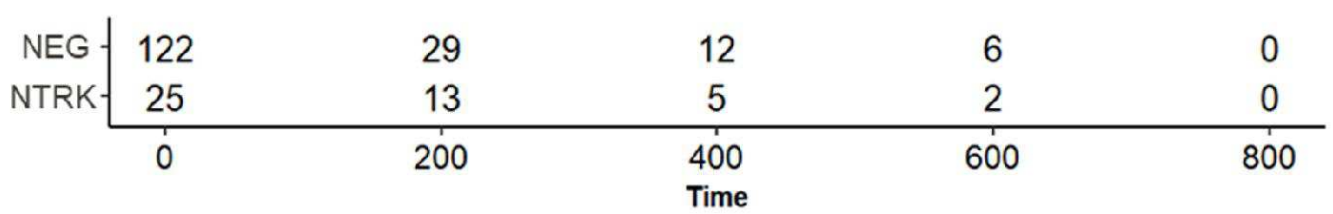


Figure 3. Kaplan-Meier PFS by NTRK alterations (NTRK fusion negative vs. NTRK1 fusion vs. NTRK3 fusion)

PFS of NTRK-fusion negative vs. NTRK1 vs. NTRK3 patients

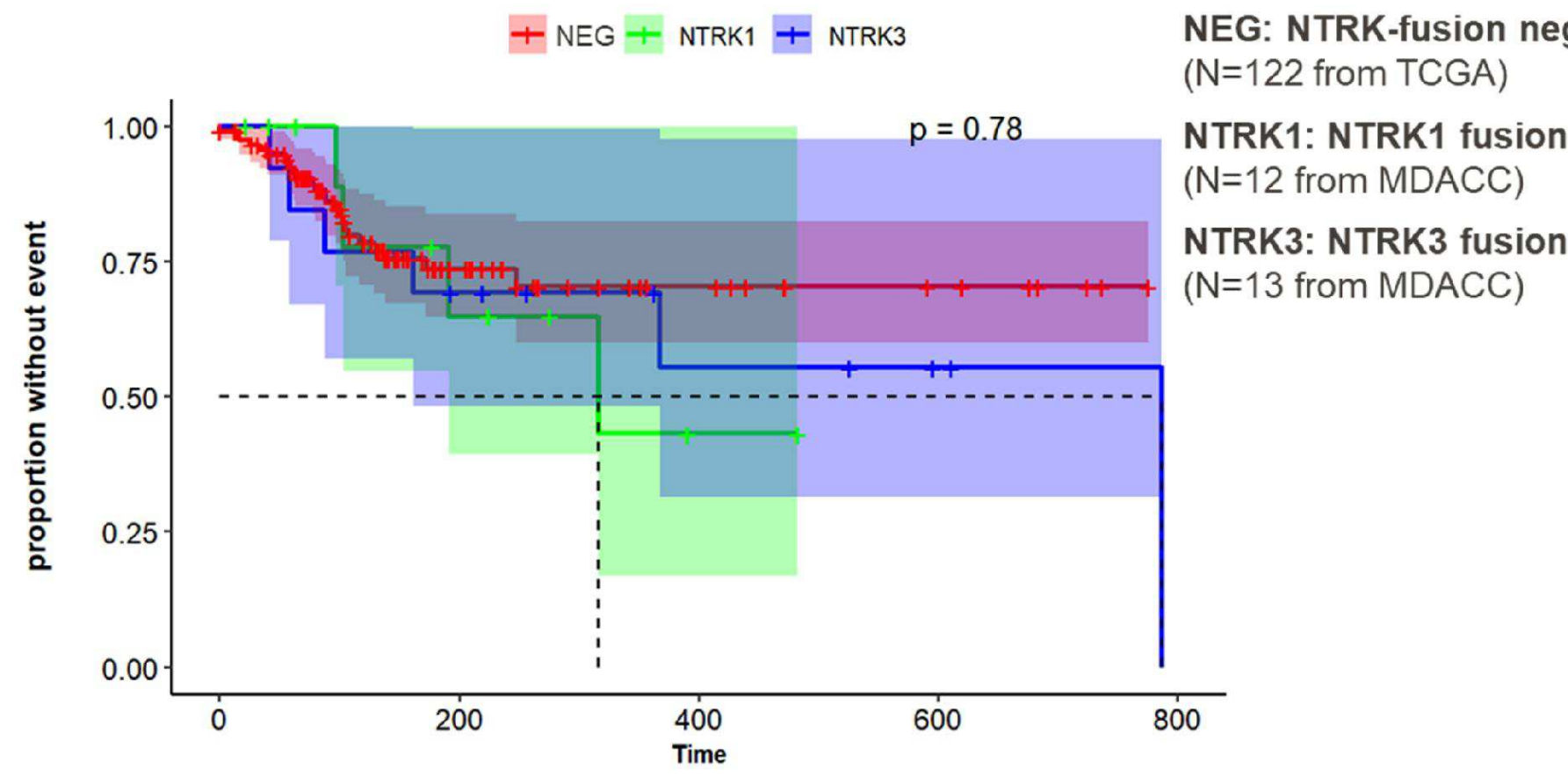

Number at risk

\begin{tabular}{|c|c|c|c|c|c|}
\hline NEG & 122 & 29 & 12 & 6 & 0 \\
\hline NTRK1 & 12 & 5 & 1 & 0 & 0 \\
\hline NTRK3 & 13 & 8 & 4 & 2 & 0 \\
\hline & 0 & 200 & $\begin{array}{l}400 \\
\text { Time }\end{array}$ & 600 & 800 \\
\hline
\end{tabular}


Table 1: Demographic and clinical characteristics of 77 MDACC patients with NTRK fusions

\begin{tabular}{|l|c|}
\hline Characteristic & Value (n=77) \\
\hline Age, median (range) & $44(1-84)$ \\
\hline Sex, number (\%) & \\
\hline Male & $34(44)$ \\
\hline Female & $43(56)$ \\
\hline Tumor type, number (\%) & \\
\hline Thyroid cancer & $34(44)$ \\
\hline Salivary gland cancer & $22(29)$ \\
\hline Sarcoma & $5(6)$ \\
\hline CNS tumor & $4(5)$ \\
\hline Colorectal cancer & $4(5)$ \\
\hline Lung cancer & $2(3)$ \\
\hline Pancreatic cancer & $2(3)$ \\
\hline Breast cancer & $2(3)$ \\
\hline Cholangiocarcinoma & $1(1)$ \\
\hline Small intestine cancer & $1(1)$ \\
\hline Stage at diagnosis, number (\%) & \\
\hline I & $13(17)$ \\
\hline II & $20(26)$ \\
\hline III & $5(6)$ \\
\hline IV & $29(38)$ \\
\hline Unknown & $10(13)$ \\
\hline
\end{tabular}


Table 2. NTRK fusions in the cohort of MDACC patients

\begin{tabular}{|l|c|}
\hline Fusion type, number (\%) & Value (n=77) \\
\hline NTRK1 & $23(30)$ \\
\hline NTRK2 & $2(3)$ \\
\hline NTRK3 & $52(67)$ \\
\hline Fusion partners, number & \\
\hline NTRK3-ETV6 & 43 \\
\hline NTRK1-TPM3 & 9 \\
\hline NTRK1-TPR & 4 \\
\hline NTRK1-IRF2BP2 & 3 \\
\hline NTRK3-EML4 & 3 \\
\hline NTRK3-SQSTM1 & 2 \\
\hline NTRK3-TFG & 2 \\
\hline NTRK1-ARHGEF2 & 1 \\
\hline NTRK1-BCAN & 1 \\
\hline NTRK1-NFASC & 1 \\
\hline NTRK1-PLEKHA6 & 1 \\
\hline NTRK1-RABGAP1L & 1 \\
\hline NTRK1-RGS7 & 1 \\
\hline NTRK1-SQSTM1 & 1 \\
\hline NTRK2-BCF & 1 \\
\hline NTRK2-BCR & 1 \\
\hline NTRK3- RBPMS & 1 \\
\hline NTRK3-GOLGA4 & 1 \\
\hline
\end{tabular}


Supplementary Data:

Supplementary Table 1. Clinical characteristics of unmatched cohort

\begin{tabular}{|c|c|c|c|}
\hline Variables & Level & TCGA & MDACC \\
\hline Total Sample Size & & 2476 & 62 \\
\hline AGE.AT.DX (median [range]) & & $62.00[16.00,90.00]$ & $44.5[4.00,84]$ \\
\hline \multirow[t]{4}{*}{ STAGE (\%) } & $\mathrm{I}$ & $1617(65.3)$ & $11(17.8)$ \\
\hline & II & $766(30.9)$ & $20(32.2)$ \\
\hline & III & $93(3.8)$ & $6(9.7)$ \\
\hline & IV & $0(0)$ & $25(40.3)$ \\
\hline \multirow[t]{2}{*}{ STAGE.2L (\%) } & I.II & $2383(96.2)$ & $31(50)$ \\
\hline & III.IV & $93(3.8)$ & $31(50)$ \\
\hline \multirow[t]{2}{*}{ SEX (\%) } & FEMALE & $1657(66.9)$ & $34(54.8)$ \\
\hline & MALE & $819(33.1)$ & $28(45.2)$ \\
\hline \multirow[t]{2}{*}{ NTRK Status (\%) } & Negative & $2466(99.6)$ & $0(0)$ \\
\hline & Positive & $10(0.4)$ & $62(100)$ \\
\hline \multirow{11}{*}{ TYPE (\%) } & BRCA & $910(36.8)$ & $1(1.6)$ \\
\hline & $\mathrm{CHOL}$ & $25(10)$ & $1(1.6)$ \\
\hline & COAD & $372(15)$ & $4(6.5)$ \\
\hline & GBM & $0(0)$ & $1(1.6)$ \\
\hline & LGG & $0(0)$ & $1(1.6)$ \\
\hline & LUAD & $375(15.1)$ & $2(3.2)$ \\
\hline & MISC & $0(0)$ & $14(22.6)$ \\
\hline & PAAD & $176(7.1)$ & $2(3.2)$ \\
\hline & SARC & $0(0)$ & $3(4.8)$ \\
\hline & STAD & $398(16.1)$ & $1(1.6)$ \\
\hline & THCA & $220(8.9)$ & $32(51.6)$ \\
\hline
\end{tabular}


Supplementary Table 2. Clinical characteristics of matched cohort II

\begin{tabular}{|c|c|c|c|}
\hline Variables & Level & TCGA & MDACC \\
\hline Total Sample Size & & 122 & 25 \\
\hline AGE.AT.DX (median [range]) & & $56.00[36.00,87.00]$ & $16.00[4.00,72.00]$ \\
\hline STAGE (\%) & I & $104(85.2)$ & $5(20)$ \\
\hline & II & $8(6.6)$ & $18(72)$ \\
\hline & III & $10(8.2)$ & $2(8)$ \\
\hline STAGE.2L (\%) & I.II & $112(91.8)$ & $23(92)$ \\
\hline & III.IV & $10(8.2)$ & $2(8)$ \\
\hline SEX (\%) & FEMALE & $115(94.3)$ & $14(56)$ \\
\hline & MALE & $7(5.7)$ & $11(44)$ \\
\hline NTRK Status (\%) & Negative & $122(100)$ & $0(0)$ \\
\hline NTRK.3L (\%) & Positive & $0(0)$ & $25(100)$ \\
\hline & Negative & $122(100)$ & $0(0)$ \\
\hline & NTRK1 & $0(0)$ & $12(48)$ \\
\hline & NTRK3 & $0(0)$ & $13(52)$ \\
\hline & BRCA & $5(4.1)$ & $2(4)$ \\
\hline & COAD & $10(8.2)$ & $1(4)$ \\
\hline & PAAD & $2(1.6)$ & $21(84)$ \\
\hline & THCA & $105(86.1)$ & \\
\hline
\end{tabular}


Supplementary Table 3: Summary of OS and PFS events in matched TCGA and MDACC cohorts

\begin{tabular}{|c|c|c|c|c|c|c|c|}
\hline \multirow{3}{*}{ TCGA code } & \multirow{3}{*}{ TCGA description } & \multicolumn{6}{|c|}{ Number of patients in matched cohort I (OS*, PFS** events) } \\
\hline & & \multicolumn{2}{|c|}{ All Patients } & \multicolumn{2}{|c|}{ MD Anderson } & \multicolumn{2}{|c|}{ TCGA } \\
\hline & & OS & PFS & OS & PFS & OS & PFS \\
\hline BRCA & Breast invasive carcinoma & $264(56)$ & $264(74)$ & $1(0)$ & $1(1)$ & $263(56)$ & $263(73)$ \\
\hline THCA & Thyroid carcinoma & $137(5)$ & $144(26)$ & $14(0)$ & $21(7)$ & $123(5)$ & $123(19)$ \\
\hline COAD & Colon adenocarcinoma & $10(2)$ & $21(12)$ & $1(0)$ & $2(1)$ & $9(2)$ & $19(11)$ \\
\hline PAAD & Pancreatic adenocarcinoma & $3(2)$ & $3(3)$ & $1(1)$ & $1(1)$ & $2(1)$ & $2(2)$ \\
\hline \multicolumn{2}{|c|}{ Total } & $414(65)$ & $432(115)$ & $17(1)$ & $25(10)$ & $397(64)$ & $407(105)$ \\
\hline \multicolumn{8}{|c|}{$\begin{array}{l}\text { *Overall Survival (OS) analysis considered patients untreated with TRK inhibitor } \\
\text { **PFS covers interval from initial diagnosis to progression on first line therapy or start of TRK inhibition for } \\
\text { treated patients }\end{array}$} \\
\hline
\end{tabular}

\begin{tabular}{|c|c|c|c|c|c|c|c|}
\hline \multirow{3}{*}{ TCGA code } & \multirow{3}{*}{ TCGA description } & \multicolumn{6}{|c|}{ Number of patients in matched cohort II (OS, PFS events) } \\
\hline & & \multicolumn{2}{|c|}{ All Patients } & \multicolumn{2}{|c|}{ MD Anderson } & \multicolumn{2}{|c|}{ TCGA } \\
\hline & & OS & PFS & OS & PFS & OS & PFS \\
\hline BRCA & Breast invasive carcinoma & NA & $6(3)$ & NA & $1(1)$ & NA & $5(2)$ \\
\hline THCA & Thyroid carcinoma & NA & $126(23)$ & NA & $21(7)$ & NA & $105(16)$ \\
\hline COAD & Colon adenocarcinoma & NA & $12(6)$ & NA & $2(1)$ & NA & $10(5)$ \\
\hline PAAD & Pancreatic adenocarcinoma & NA & $3(3)$ & NA & $1(1)$ & NA & $2(2)$ \\
\hline & Total & NA & $147(35)$ & NA & $25(10)$ & $\mathbf{N A}$ & $122(25)$ \\
\hline
\end{tabular}


Supplementary Table 4: Summary of variables

\begin{tabular}{|c|c|c|}
\hline Variable & Description & Level \\
\hline NTRK & Presence of NTRK fusion & $\begin{array}{l}\text { NEG = TCGA patients without NTRK } \\
\text { fusion } \\
\text { NTRK = MDACC patients with NTRK } \\
\text { fusion }\end{array}$ \\
\hline STAGE & Pathological stage at initial diagnosis & I; II; III; IV \\
\hline STAGE.2L & $\begin{array}{l}\text { Pathological stage at initial diagnosis } \\
\text { (binary) }\end{array}$ & I.II; III.IV \\
\hline WEEKS_OS & Duration from diagnosis to death & $\mathrm{N} / \mathrm{A}$ \\
\hline WEEKS_PFS & $\begin{array}{l}\text { Duration from diagnosis to death, } \\
\text { progression, } \\
\text { or start of NTRK therapy }\end{array}$ & N/A \\
\hline AGE.AT.DX & Age at disease diagnosis & \\
\hline NTRK.3L & $\begin{array}{l}\text { Presence of NTRK1, NTRK3, versus } \\
\text { Absent }\end{array}$ & MIX; NTRK1; NTRK3 \\
\hline SEX & Sex & FEMALE; MALE \\
\hline TYPE & TCGA histology code & $\begin{array}{l}\text { BRCA; CHOL; COAD; GBM; LGG } \\
\text { LUAD; MISC; PAAD; SARC; STAD; } \\
\text { THCA }\end{array}$ \\
\hline
\end{tabular}

